\title{
Metaheuristics methods for The VRP in Home Health Care by minimizing fuel consumption for environmental gain
}

\author{
Haitam Ettazi ${ }^{1}$, Najat Rafalia $^{1}$, and Jaafar Abouchabaka ${ }^{1}$ \\ ${ }^{1}$ Faculty of Science, Ibn Tofail University, Kenitra, Morocco
}

\begin{abstract}
Nowadays, the international society began to see clearly the impact of neglecting the environmental aspect in our lives, especially the issue of global warming. This paper aims in the first degree to minimize the carbon emission by vehicles and in doing so help to reduce the pollution ratio that is increasing day by day. In the home care sector, a patient requires from a health structure offering home care services one or many services to be done at home. For this purpose a health staff is mobilized to provide these services and must be done optimally. Many papers dealing with this kind of problems are suggesting that the way to optimize the problem encountered efficiently is to use the resources put in place to minimize the total travelled distance, but in this perspective, the study prioritizes the reduction and minimizing the fuel consumption. To answer all this questions, an exploration and an investigation of the problems addressed in the literature is performed, after that a mathematical formulation of the problem and two metaheuristics methods are proposed to resolve this problem. In the end, a comparison of the results of the two methods is presented.
\end{abstract}

\section{Introduction}

The transportation sector is estimated to generate for about $23 \%$ of the $\mathrm{CO}_{2}$ emission where the road sector is responsible for about $75 \%$ of it, declares the report of International Energy Agency (IEA). This situation arises the need to find a way to reduce the $\mathrm{CO}_{2}$ emissions by the transportation, in our case the road sector.

In the home health care area, a patient that does not need any more to stay at hospital is suggested to leave their spot to other needy patients. Therefore theses structures propose a set of services and cares required by this type of patients to be performed at home. For this purpose, a system of transportation is needed to fulfill this task by taking into consideration many factors such as the patient's time windows, priority and precedence if a patient need more than one service at a time or successively (one after another in a specific order). These variables require an adaptive system to be put in order to achieve all this heavy and predetermined tasks in an efficient manner.

The home health care sector as becoming worldwide especially in this era of COVID-19 [1], it is very important to cite that pandemic prioritized the necessity of giving cares to patients in their homes. This type of services such as assistance, medical care, bathing, cleaning is becoming more required by old people or disabled one and need a proper preparation in order to allocate staff to perform them without making the patients come to structures outside their homes and in doing so, it is best to optimize all the resources to the success of this matter. A fine and flexible coordination must be established for material resources and the caregivers in order to generate an optimized planning which increase the quality of home health care and decrease the overall costs either costs related to fees and charges by the structures providing care or costs dealing with environmental issues such as the emission of $\mathrm{CO}_{2}$ gas.

In this paper, the study is bound to work with the classical vehicle routing problem to tackle some of the characteristics linked to the home health care services. The main objective with the vehicle routing problem is to offer a set of routes to be taken by a fleet of vehicles usually associated in our case to caregivers to visit inside a predetermined geographical area a number of customers corresponding to patients who have preferences such as time windows, precedence... This study emerges into a new aspect not only dealing with the logistical aspect related to providing cares to patients only, but also deal with the reduction of fuel consumption by vehicles associated to caregivers. The problems encountered in home health care sector always have timing constraints associated with it, the paper then add on the constraint of fuel consumption which give us in the end a variant of the vehicle routing problem called VRPTW-SPFC for Vehicle Routing Problem With Time Windows, Synchronization, Precedence and Fuel Consumption constraints. The Structure of this paper 
will be rather logical and make a continuity of the problem itself with section 2 dealing with literature review, the problem presentation will be addressed in section3, the mathematical formulation of the problem using the Mixed Integer Linear Programming(MILP) will have its own section numbered 4 , The 5 th presents the approached method, and the section 6 will show the results of the methods applied in the precedent section, a discussion section 7 and to finish section 8 is dedicated to the conclusion and possible perspectives of this work.

\section{Literature review}

Among the considered NP-Hard problems, the vehicle routing problem is one of the most studied within the field of Operational Research in the last 20 years. To put a clear definition to the vehicle routing problem that was firstly introduced by [2] is to construct a delivery path or route with the least cost possible from a depot to a set of scattered locations (customers) according to a number of constraints that should be respected. The resolution approaches consist of both exact and heuristics methods. The metaheuristic method is considered to be the most efficient until which is found in [3] that can solve up to 26 vehicle routing problem variants. The core study of this paper involves the allocation and planning of various resources such as vehicles, caregivers, material and so on to a number of requested demands made by a set of patients dispersed in a geographical zone to obtain specific and predefined services. From that angle, many constraints arise such as time windows determined by the patient, patient's preferences toward caregivers, synchronization of services... The best methods used until now for many years to solve such prerequisites is heuristics and metaheuristics approaches such as proposed by [4-7]. In order to dig further into the literature, it is mandatory to look for the most objectives (cost, preferences, waiting time...) considered in those studies. In the vehicle routing problem under the context of home health care, the total travel time, the number of vehicles required, the total travelled distance by the vehicles, the preferences of patients toward caregivers are the most used objectives often studied separately and can be found very rarely attached together as a multiple objective study.

The idea laid under the logistical issue such as transportation in the home care can be divided into two categories, allocation and planning issues as well as pickup and delivery issues.

For the sake of the study, the paper ought to separate this section in three subsections, the first one is dedicated to references and studies already made by many authors on this subject especially those related to vehicle routing problem in home health care sector, the second will be an extension of contributing references related to the aspect of vehicle routing problem with precedence and/or synchronization and papers based on fuel consumption minimization and the last subsection describes the highlights of the contribution made in this paper.

\subsection{VRP in Home Health Care Sector}

In the context of Health Systems, the logistics aspect was first showed by [8-10] on the door to door transportation for serving the disabled and/or the elderly people. The growing rate of elderly people worldwide have led the authorities to put down a system called DARP which translate to Dial-A-Ride to aid people suffering from disabilities in any sort. From this perspective, a specific variant of DARP was developed by $[11,12]$ who aims to send a special team with adequate and specific qualifications to the patient's home to support them in whatever help they need (bath, dress, help to move...) before the arrival of the resource responsible to transport them to the hospital or a care structure. To ensure the success of this operation, a fine coordination is required by nursing assistant and transporters, this scenario have led researchers to introduce constraints such as synchronization constraints in order to ensure the respect of priority between visits. In the same category, patient's demands emerges a new constraint which allows them to choose a time windows in which they are ready to receive the care staff in their homes. [13] propose and introduced this aspect of time windows in the DARP for better planning, but does not consider the condition of emergency and only concerns the minimization of the costs generated by the transportation of the patient. A study well known in the literature have extended the Dial-A-Ride problem into the HHCP (Home Health Care Problem) conducted by [14] in which the core idea is to assign caregiver's workers to provide a certain number of cares requested by customers (patients) in their homes. The study illustrates two resolution methods, the first one was an exact method by proposing a mathematical model with integer mixed variables (MILP) and the second is a heuristic method. The numerical results were applied on 4 nurses and 10 patients. After this first step, many papers were conducted in the same way and various optimization tools were applied to deal with it.

In Home Health Care (HHC) context, various fields were studied considering the logistical issues. Two broad categories were distinguished among the rest and deemed to be the most important aspect in order to optimize the problem at its most. The planning and allocation issues as well as pickup and delivery. The best and most simple way to understand the matter in general is that a caregiver is assigned to a patient in order to do a task required by this latter. This caregiver is defined by a certain set of skills (medical qualification, gender, age, specialty, language...), a defined amount of work hours with a begin and an end from the care structure (depot). Each task or care is characterized by a time windows, duration and skill. 
The issue of attributing visits to personal caregivers and the visits planning was studied in [15] where they introduced a MILP method and a Tabu search to resolve the problem and output reasonable and good quality solutions.

The same problem was expressed in [16] but as a partitioning problem aiming to minimizing the time of transportation and the non satisfaction of patient's toward caregivers.

[17] introduced the constraint of time windows which led to the VRPTW (Vehicle Routing Problem with Tile Windows) and solved with a MILP formulation and a metaheuristics based on variable neighboring search to reduce the nurses movement's time and to increase the ratio patients/nurses of satisfaction.

To solve the VRPTW applied in the home health care, [18] used a PSO (Particle Swarm Optimization) in order to minimize the total travelled distance by caregivers respecting the time windows of visits and the capacity of vehicles.

Many studies propose only a single objective optimization, but in [19] opted for a multi-objective optimization by proposing a mathematical formulation and two metaheuristics based on population (Ant Colony Optimization) and a multi-objective genetic algorithm.

$[20,21]$ consider the notion of availability of patient at home to establish a coordinated visit between caregivers to perform several cares either simultaneously or in a defined order bound by priority which fall down under synchronization aspect. The objectives were to minimize the cost of travel by caregivers and maximize the preferences of patients toward caregivers. A Branch and Bound algorithm was proposed as a resolution method by the authors.

A MILP for modeling the problem for scheduling the visits to patients and assigning the tasks to caregivers was established by [22] to reduce the waiting times of the patients.

A linear programming with mixed variables (MILP) was proposed by [22] in which an important optimization criterion in the improvement of home care structures has been introduced. In addition to minimizing the cost travelling, authors were attracted in minimizing and shortening the patients waiting time, especially those who requested multiple synchronized visits.

Conducting an heuristic in two stages were proposed by $[23,24]$ to assign routes to caregivers firstly by defining and generating a set of routes then introduce synchronization constraints to attain feasible solutions.

[25] developed a new mathematical formulation to run away from nodes duplication. An ILS (Iterated Local Search) was coded and tested on instances relatively small.

[26] prioritize the maximization of patient satisfaction and minimization of travel cost by applying a metaheuristic on VNS (Variable Neighborhood Search).
More recently, in the Home Health Care Routing and Scheduling Problem (HHCRSP) which count as an extension of the VRPTW with multiple availability periods of customers (Patients) considering soft/flexible time windows, [27] propose a mathematical formulation to reduce both the total waiting time of caregivers and the total penalized tardiness and earliness of the operations if services.

The authors in [7] added the synchronization aspect to the VRPTW problem where a demand made by a patient can include more than one visit either simultaneously or in a given order (precedence), a MILP, a Greedy heuristic and three metaheuristics was developed to minimize both the total travel cost and the non-preferences of patients toward caregivers.

The next thing to specify in our study is the Pickup and Delivery problem in the hole health care services where a patient have to be picked up and/or dropped off from a certain location to another as explained in [13]. The authors in [4] were interested in the aspect of delivering meals to disabled and/or elderly patients to their homes and combined various variants of problem encountered in the health system. On this ground, patients are clustered in zones and caregivers in teams. Caregivers have a certain set of skills and a certain amount of hours to work per day and must perform different tasks within the day. The goal behind is to maximize to the most the hours of visits and the preferences of staff.

[28] present the same problem as explained before as alternation which the delivery considers medication and medical devices instead of meals. It considers picking up and delivering biological samples and/or medical devices to patient's homes taking into consideration time windows constraints and are planned in simultaneous way. This study aims to minimize unsatisfied demands where an exact method based on columns generation was proposed to resolve the problem.

\subsection{VRP with synchronization constraints}

The important notion that arises the need to study such a problem in many various ways is its timing aspect which enforces to find feasible practices to gain even a small amount of time to save the life of the needy person or in the least case serve them in an efficient manner with the least cost possible. The challenge behind synchronization is to satisfy the requests of patients whatever it takes by scheduling the tasks given to caregivers either simultaneously or successively.

[29] proposes an interdependence related to routes performed by caregivers along with time windows linked to patients, and suggested this interdependence as a classification in synchronization of operations, and more than that [25] clustered this synchronization into two clusters which lead to two types of synchronization: 
1. Precedence synchronization: Where a patient requires more than one service which lead to schedule two or more visits ( When it is mandatory to perform a task before another for example preparing a patient by cleaning and clothing him before transporting him to another location).

2. Simultaneous synchronization: Where a patient request a service which cannot be done by only one caregiver therefore the staff in charge of doing the task must be composed of two or more caregivers (for example bathing a patient require the intervention of two caregivers).

Before moving on to the fuel consumption aspect, the study makes a tour on the literature dealing with the synchronization nature in the vehicle routing problem in the home health care.

[30] work on the household waste collection problem but introducing synchronization constraints. their goal was to reduce the total duration of journeys where the trucks used were separated in two kinds, small (satellites) authorized to drive on any type of road and large (compactors) where it can only travel on narrow and small streets due to their width. The aspect of synchronization occurs when a satellite must discharge the compactors from its contents so the synchronization happens in that case when the last two must arrive at the same time (simultaneously). The authors used a heuristic based on a local search procedure with a variable descent called VND (Variable Neighborhood Descent) where the objective function is to minimize the movements cost of the trucks.

[31] worked on the arc routing problem with synchronization constraints to perform snow removal in Canada. The aim of the modeling proposed was to determine the set of routes which allow the minimization of the duration of the longest route simultaneously with the snow removal operation. the proposed method was a MILP and a heuristic based on the ALNS (Adaptive Large Neighborhood Search) metaheuristic.

A vehicle routing problem with time windows for home health care routing and scheduling problem and synchronized visits to optimize the sequence visits to homes and execution visits by personal's assignment was proposed by [32]. The method used is a genetic algorithm alongside a hybrid genetic algorithm in order to reduce the difference in service time between various vehicles for workload balance.

In the exploration of the papers found in the literature it is hardly to find a paper dealing with the vehicle routing problem in home health care sector in order to optimize and reduce the fuel consumption. Therefore all papers touching the field of home health care sector considers the optimization by reducing the total travel distance, so for this paper, it considers the minimization of fuel consumption in the home health care sector alongside all the constraints illustrated above and compare it to results found in various papers and showing that reducing the $\mathrm{CO}_{2}$ emission is more effective and efficient for both cost issues and environmental issues than the total travelling distance.

To illustrate the notion of reduction of fuel consumption, this paper cites some of the works dealing with this aspect in various fields and how authors deal with it in the logistical world.

[33] worked on the ship routing problem to make a decision optimally the type of ships and their numbers. He concluded that the fuel consumption is based on the distance sailed and the type of ship to choose which ship to choose. In the same line, [34] decided that the nautical distance travelled in miles depends on the fuel consumption. [35,36] both used optimization software decides that fuel consumption is based on transportation distance. The fuel consumption is usually connected to the travelled distance either in mile or in Kilometer where [37] worked with an optimization software for optimization routes in solid waste collection where he declares that the fuel consumption relied on the distance travelled, vehicle load and road gradient.

These works clearly show that fuel consumption are based on different criteria mostly the distance travelled and the vehicle load as long as the type of road taken by the vehicles.

\subsection{Contribution}

Based on the literature review, the works related to vehicle routing problem in home health care sector considering both simultaneous synchronization and precedence constraints was made by duplicating nodes attached to patients that lead to an increase in the size of the network. The mathematical modeling was modeled by many of the papers seen above using linear solver such as CPLEX and exact method, it is found that metaheuristics based on local search have been explored and tested in that line. This study concluded that these approaches can only lead to a feasible solution for small instances and output degrading and not so encouraging results for bigger ones. As for the objectives considered in those papers, the total travelled distance has the highest rate of use with almost no study considered the reduction of fuel consumption especially in the home health care sector. Our contribution is as follows:

- Merge the simultaneous and precedence synchronization in one model by duplicating nodes and testing it for bigger instances.

- Implicate many constraints such as staff preferences, synchronization with both types, and patient's preferences toward caregivers.

- Consider the objective of minimizing the fuel consumption as opposed to most of papers dealing with home health care and compare it with the 
common objective function usually found in the literature.

- Develop and propose an efficient metaheuristic in order to obtain and solve rather large instances in existing benchmarks.

\subsection{Methodology}

Before jumping into the problem description and the proposed approach, the methodology used to resolve the VRPTW-SPFC is as follows:

1. Define the variables and parameters of the problem and the objective function to solve.

2. Propose a mathematical formulation of the problem using the MILP, the equations are solved using to CPLEX Solver.

3. Propose and apply two metaheuristics; the TS and the VNS on an instance found in the literature.

4. Compare the results obtained.

\section{Problem Description}

This paper addresses a variant of the vehicle routing problem with time windows, precedence and synchronization constraints for reducing fuel consumption in the Home Health Care System.

For the simplification and generalization, the paper considers that the capacity of vehicles is null because it only transports medical staff and rather not heavy medical devices.

The VRPTW-SPFC is considered as a graph $\mathrm{G}=(\mathrm{V}$, E) where $\mathrm{V}$ is a node-set $\mathrm{V}=\mathrm{NUD}$ where $\mathrm{N}=\{1 \ldots \mathrm{n}\}$ corresponds to a group of patients (customers) and $\mathrm{D}=$ $\{c, f\}$ are nodes where $\mathrm{c}$ is the initial depot (center) and $\mathrm{f}$ the final depot. $M$ denotes as an arc (margin) set where each arc $\mathrm{m}=\left(c_{i}, c_{j}\right): c_{i} \neq c_{j} \in M, i \neq j$ is linked to a fuel consumption $\mathrm{f}_{\mathrm{ij}}$ and a travel distance $\mathrm{d}_{\mathrm{ij}}$ and a travel duration $\mathrm{T}_{\mathrm{ij}} . \mathrm{K}$ is the set of vehicles available at the initial depot (center) c and for each $\mathrm{k} \in \mathrm{K}$ an availability time window $\left[\alpha_{k}, \beta_{k}\right]$ where a vehicle $\mathrm{k}$ can only leave the depot $\mathrm{c}$ at $\alpha_{k}$ and must return to the final depot $\mathrm{f}$ at $\beta_{k}$. S denotes as a set of services where the initial depot (center) $\mathrm{c}$ provides multiple services $\mathrm{S}=\{1 \ldots \mathrm{s}\}$ to patients (customers). A patient $\mathrm{i}$ demands a subset of services realized by a set $\mathrm{S}_{\mathrm{i}}=\left\{\mathrm{s} \in \mathrm{S}: \mathrm{e}_{\mathrm{is}}=1\right\}$ where $e_{i s}$ matches 1 if a patient $i$ requires services $\in S$, and 0 if not. Each service $\mathrm{s}$ is given to a patient $\mathrm{i}$ and has a time window $\left[a_{i}, b_{i}\right]$ and a time duration $d_{i s}$; where $a_{i}$ is the earliest service start time and $b_{i}$ the latest service start time specified for a patient i. To assure ensure the preference of each vehicle $\mathrm{k}$, a number $\operatorname{Pr} f_{i k}$ is given to the vehicle $\mathrm{k}$ and marks the non-preference of patient (customer) i to the caregiver (vehicle) $\mathrm{k}$.
For the generalization of this study, $\mathrm{k} \in \mathrm{K}$ provides an unique service s. $y_{k s}$ is equal to 1 if the vehicle $\mathrm{k}$ provides the service $\mathrm{s}, 0$ otherwise $(\forall \mathrm{k} \in \mathrm{K}, \forall \mathrm{s} \in \mathrm{S})$.

For customers (patients) whom are demanding more than one service either in a specific order or simultaneously, the authors define for each patient $i \in \mathrm{N}$ a slot $_{i s r}$ as the time separating the time start of service $\mathrm{s}$ and service $r$ demanded by patient $i$ where services $s$ and $r$ have to be provided in a given order (service $\mathrm{s}$ before service $r$ ).

When a synchronized service is demanded by a patient requiring two or more caregivers at an exact order (precedence), this study defines a gap $_{i s r}$ (the time of starting of service $s$ and $r$ ensured to patient $i$ ), as for the simultaneous case and according to [38] the authors put gap $_{i s r}=$ gap $_{\text {irs }}$.

This problem formulation considers the minimization of the total carbon emission produced by all the vehicles and the sum of non-preferences of patients towards caregivers.

$[7,25]$ have conducted similar studies but did not consider as the objective the reduction of fuel consumption. This kind of problem is known to be an NP-hard problem. For the first resolution, a Mixed Integer Linear Program (MILP) is used considering the binary and real variable decision respectively as follows:

- $\mathrm{x}_{i j k}=1$, if $\mathrm{k} \in \mathrm{K}$ travel from i to $\mathrm{j}, 0$ otherwise

- $s_{i k}$ infers to the start time of service at node $\mathrm{i}$ if customer (patient) $\mathrm{i}$ is visited by vehicle (caregiver) $\mathrm{k}$.

To generalize the constraints of time in this formulation, the paper presumes that all the requested services by patients are asked independently from the initial depot (center) $\mathrm{c}$ and their duration is $0\left(\mathrm{D}_{c s}=0\right.$, $\forall \mathrm{s} \in \mathrm{S}$ ).

As for the fuel consumption, the relationship between the fuel consumption and the travelled distance is considered as linear. The rate $r f$ of fuel consumption is put as a fixed value. So to compute and calculate the fuel consumption by vehicle $\mathrm{k}$ in $\operatorname{arc}\left(c_{i}, c_{j}\right)$ put $\mathrm{f}_{\mathrm{ij}}=\mathrm{rf} . \mathrm{d}_{\mathrm{ij}}$.

The study adds a factor called $C C F$ called Carbon emission Conversion Factor which is according to [39], a vehicle produces in general $0.2 \mathrm{~kg}$ of $\mathrm{CO}_{2}$ per mile of travelled distance by a vehicle, with this perspective, the papers assumes that $C C F=0.2 \mathrm{~kg}$ per miles.

The remaining parameters to complete the description of variables are $\mathrm{T}$ as the tank capacity of vehicles and $f_{i}$ the fuel remaining after visiting patient $\mathrm{i}$.

\section{Mathematical formulation}

The MILP corresponding to our formulation is as described:

$$
\begin{aligned}
& \quad \min \sum_{i \epsilon V \backslash\{f\}} \sum_{j \epsilon V \backslash\{c\}} \sum_{k \epsilon K} d_{i j} x_{i j k} \times \mathrm{CCF}+ \\
& \sum_{i \in N} \sum_{j \epsilon V \backslash\{c\}} \sum_{k \epsilon K} \operatorname{Prf}_{i k} x_{i j k}
\end{aligned}
$$


Subject to:

$$
\begin{aligned}
& \forall \mathrm{k} \in \mathrm{K}, \sum_{\mathrm{j} \in \mathrm{N}} \mathrm{x}_{\mathrm{cjk}}=1 \\
& \forall \mathrm{k} \in \mathrm{K}, \sum_{\mathrm{j} \in \mathrm{N}} \mathrm{X}_{\mathrm{ifk}}=1 \\
& \forall \mathrm{h} \in \mathrm{N}, \forall \mathrm{k} \in \mathrm{K}, \\
& \sum_{\mathrm{i} \epsilon \bigvee \backslash\{f\}} \mathrm{X}_{\mathrm{ihk}}=\sum_{\mathrm{j} \in \mathrm{V} \backslash\{\mathrm{c}\}} \mathrm{X}_{\mathrm{hjk}} \\
& \forall \mathrm{i} \in \mathrm{N}, \forall \mathrm{s} \in \mathrm{S} \text {, } \\
& \sum_{\mathrm{j} \in \mathrm{V} \backslash\{\mathrm{c}\}} \quad \sum_{\mathrm{k} \in \mathrm{K}_{\mathrm{s}}} \mathrm{x}_{\mathrm{ijk}}=\mathrm{e}_{\mathrm{is}} \\
& \forall \mathrm{i}, \mathrm{j} \in \mathrm{V}, \forall \mathrm{s} \in \mathrm{S}: \mathrm{s} \in \mathrm{S}_{\mathrm{i}} \cup \mathrm{S}_{\mathrm{j}}, \forall \mathrm{k} \in \mathrm{K}_{\mathrm{s}}, \\
& s t_{i k}+\left(T_{i j}+D_{i s}\right) x_{i j k} \leq s t_{j k}+b_{i}\left(1-x_{i j k}\right) \\
& \forall \mathrm{i} \in \mathrm{N}, \forall \mathrm{s} \in \mathrm{S}_{\mathrm{i}}, \forall \mathrm{k} \in \mathrm{K}_{\mathrm{s}}, \\
& \mathrm{a}_{\mathrm{i}} \sum_{\mathrm{j} \in \mathrm{N}} \mathrm{x}_{\mathrm{ijk}} \leq \mathrm{st} \mathrm{t}_{\mathrm{ik}} \leq \mathrm{b}_{\mathrm{i}} \sum_{\mathrm{j} \in \mathrm{N}} \mathrm{x}_{\mathrm{ijk}}
\end{aligned}
$$

$$
\forall \mathrm{k} \in \mathrm{K}, \quad \alpha_{\mathrm{k}} \leq \mathrm{st}_{\mathrm{ck}} \leq \beta_{\mathrm{k}}
$$

$$
\forall \mathrm{k} \in \mathrm{K}, \alpha_{\mathrm{k}} \leq \mathrm{st}_{\mathrm{fk}} \leq \beta_{\mathrm{k}}
$$$$
\forall i \in N, \forall s, r \in S_{i}, r \neq s,
$$$$
\sum_{\mathrm{k} \in \mathrm{K}_{\mathrm{r}}} s t_{\mathrm{ik}}-\sum_{\mathrm{k} \in \mathrm{K}_{\mathrm{s}}} s t_{\mathrm{ik}} \leq \operatorname{gap}_{\mathrm{isr}}
$$$$
\forall \mathrm{i} \in \mathrm{N}, \forall \mathrm{s}, \mathrm{r} \in \mathrm{S}_{\mathrm{i}}, \mathrm{r} \neq \mathrm{s},
$$$$
\sum_{\mathrm{k} \in \mathrm{K}_{\mathrm{r}}} s t_{\mathrm{ik}}-\sum_{\mathrm{k} \in \mathrm{K}_{\mathrm{s}}} s t_{\mathrm{ik}} \leq-\mathrm{gap}_{\mathrm{isr}}
$$$$
\forall i \in N, \forall s, r \in S_{i}, r \neq s,
$$$$
\sum_{\mathrm{k} \in \mathrm{K}_{\mathrm{r}}} \mathrm{st}_{\mathrm{ik}}-\sum_{\mathrm{k} \in \mathrm{K}_{\mathrm{s}}} \mathrm{st} \mathrm{ik}_{\mathrm{k}} \leq \mathrm{gap}_{\mathrm{isr}}-\mathrm{X} . \mathrm{u}_{\mathrm{i}}
$$

$\forall i \in N, \forall s, r \in S_{i}, r \neq s$,

$$
\sum_{k \in K_{r}} s t_{i k}-\sum_{k \in K_{S}} s t_{i k} \leq-g a p_{i s r}+\mathrm{X} .\left(1-\mathrm{u}_{\mathrm{i}}\right)(13)
$$

$$
\forall i, j \in N
$$$$
f_{j} \leq f_{i}-r \times d_{i j} \sum_{k \in K} x_{i j k}+T\left(1-\sum_{k \in K} x_{i j k}\right)(
$$$$
\forall i, j \in N \quad f_{j} \leq T-r \times d_{i j} \sum_{k \in K} x_{i j k}
$$$$
\forall i, j \in V \quad f_{i} \geq r \times d_{i j} \sum_{k \in K} x_{i j k}
$$

$$
\forall i, j \in V, \forall k \in K, \quad x_{i j k} \in\{0,1\}
$$

$\forall i \in V, \forall k \in K, \quad g_{i k}, g_{i k}^{\prime} \in\{0,1\}$

$$
\forall i \in V, \forall k \in K, \quad s t_{i k} \in\{0,1\}
$$

The objective function (1) is to minimize the total travelling time and the sum of non-preferences. Constraints (2) and (3) ensure that each vehicle must leave initial depot and return to it. Constraint (4) secures the continuity and constancy of the routes while constraint (5) ensures that the demands of customers (patients) are provided and executed. Scheduling permitting the consistency between the durations of visits is maintained by constraint (6). Constraints (7), (8), (9) guarantee the respect of patients, caregivers time windows. Constraints (10), (11), (12) and (13) define the constraints of synchronization between the beginning time of the services required by the patients who is requesting more than one services. Constraint (14) calculate the fuel consumption in the state where a vehicle is travelling between two patients while constraint (15) calculates the fuel consumption of vehicles from the initial depot to the patient's home and finally constraint (16) guarantee that the fuel remaining will assure the travel until the next destination either a patient's home or the depot . As for constraints (17), (18) and (19) define the nature of the decision variables.

\section{Resolution Approach}

The approach used in this paper is based on problem decomposition into a daily routing problem. With this practice, this study could have a prevision on the reduction of fuel consumption for any period of time. In first place, the paper has to determine the overall routes for each caregiver (vehicle) for a period of one day. The planning considered for a wide period going from one week to a month in order to have a better view of the results obtained. For all those parameters, the authors of this study opted to work on a basis of one day in order to attain a charge balance. The experiments made to solve the studied problem will be conducted by using a linear solver with CPLEX which demonstrated to have a limitation and cannot produce good and feasible results for bigger instances but output relatively good one for small instances. For the purpose of testing this paradigm on bigger instances, the authors were faced and forced to find an alternative method to reach the desired results for big instances and by going in line with what was said, the better solution was to choose an approached method in order to satisfy all the demands requested by the patients. This led us to choose the option of metaheuristics and in our case the use of two methods: the Tabu Search algorithm and the VNS (Variable Neighborhood Search) algorithm are made. Before moving on to how this study applied these algorithms on the instance supplied, firstly the paper introduces and gives a description of the neighborhood operators which are the use base and without those operators the authors cannot develop and apply the two methods on the problem.

\subsection{Neighborhood operators}

Tabu Search algorithm firstly introduced by [40] and the algorithm of variable neighborhood are based on two 
types of operators, the insertion operator and the swap operator.

To show the utility of each one of them and the difference between them, the insertion operator posses three possible moves, the first move is when a demand which belongs already to a known route and can be inserted into another, the second is when a sub route has been decided with a calculated fuel consumption higher than another it can be inserted in the route with the least fuel consumption rate and finally a demand belonging to a route can be omitted from it and inserted to set of demands waiting for a route.

The second type of the neighborhood operator is the swap operator. this one posses two types of movements. A demand belonging to a set of requests of services from patients can be swapped as explained in [41] in order to construct a neighborhood solution by doing the permutation, the other type of swap procedure is to swap a route with another one belonging to the same route or to different one. The idea lying behind the swap operator is to avoid to be trapped into a local optimum to the point that the swap can be operated randomly.

\subsection{Algorithms}

\subsubsection{Initial Solution}

The first step to consider before using the methods suggested in generating an initial solution. This latter is constructed and generated by applying the patient's demands to an insertion procedure into routes one by one considering the best position to do the insertion, with regards the precedence constraint. In the case of our study, the precedence between demands of clients is valued according the time windows and the availability of the staff (caregivers) and to the type of synchronization desired and approved by the patient. After finishing a visit the fuel consumption rate is calculated and added to the overall fuel consumption all over the day. When a demand for some reason cannot be served, the request is placed then into a list of unresolved demands and reported.

\subsubsection{Tabu Search}

The classical Tabu Search (TS) algorithm as defined by [40] which introduced a process that is iterative in order to make an exploration of the space of solutions by moving between one solution to the neighboring one.[42] transform the routine of optimization in general from being trapped in a local optima to escape effectively from it.

TS have rather a classical structure: from the initial solution generated, the TS algorithm moves in the space of current solution to another by exploring the neighboring without falling under the solutions considered as taboo until encountering and meeting the stop condition. In this study, the study considers the insertion operator for neighborhood search. The TS algorithm has a very special list called the tabu list where demands have been recently placed. The stop condition in our case is either the emptiness of the fuel tank or a predefined max iteration corresponding to the tabu list size.

\subsubsection{Variable Neighborhood Search (VNS)}

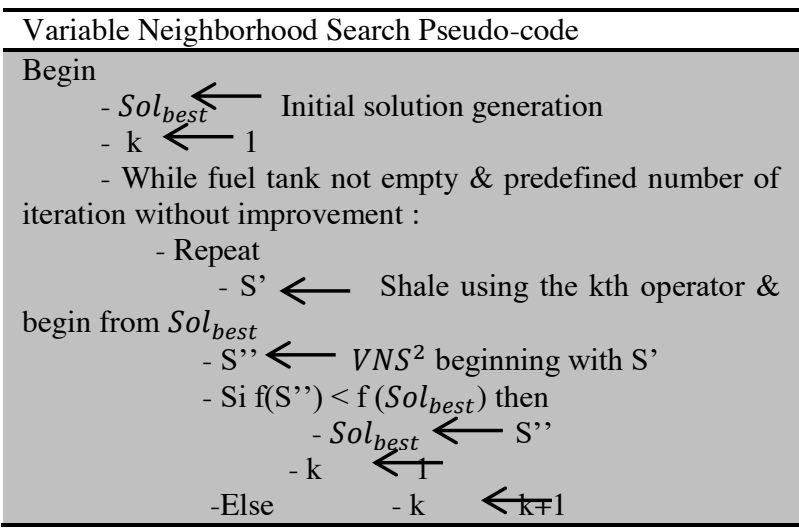

\section{Numerical results}

To illustrate the methods used to solve the problem, this paper validates the methods on a set of home health care instances by [21]. The algorithms were implemented in Python 3.8 for metaheuristics and CPLEX Studio version 12.10 for the exact method. The tests were realized on a2.4 GHz Intel Core (TM i7-5500 CPU) machine with $8 \mathrm{~GB}$ of RAM, working on Windows 10 Home. The instances used are composed of 3 groups of instances, the first is a set relatively small and composed from 18 customers (patients) and 4 vehicles (caregivers), the second from 45 customers (patients) with the use of 10 vehicles (caregivers) and the last one is composed from 80 patients (customers) and 16 vehicles (caregivers).

As for dealing with the synchronization issue, authors choose to work with a range of numbers associated with synchronization going from two to four. For the sake of generalization, this study assumes that the Tank capacity cannot be emptied after visiting the patient. The widths of time windows are defined as small, medium and large. In this paper, the limit of number of requested services cannot exceed two services at the same time.

[43] adopted a uniformly located destination in order to objectively judge the fuel consumption, in this case the authors adapt the set used to operate and compute the fuel consumption in our field of study which is the home health care sector. 4 set are used to define the speed level of each route of transportation. This paper assumes that speed level will be chosen randomly in this paper. Table 1 describes the ratio of transportation speed level by percentage in each set (High speed, Medium speed and Low speed). 
Table 1. Data set of transportation speed

\begin{tabular}{|c|c|c|c|}
\hline \multicolumn{4}{|l|}{ Ratio of transportation speed level } \\
\hline Set & $\begin{array}{c}\text { High Speed } \\
\%\end{array}$ & $\begin{array}{c}\text { medium } \\
\text { speed \% }\end{array}$ & Low speed \% \\
\hline 1 & 33.33 & 33.33 & 33.33 \\
\hline 2 & 40.00 & 40.00 & 40.00 \\
\hline 3 & 20.00 & 40.00 & 20.00 \\
\hline 4 & 40.00 & 20.00 & 40.00 \\
\hline
\end{tabular}

Table 2. MILP Solutions

\begin{tabular}{|c|c|c|}
\hline Instance & Objective & CPU(ms) \\
\hline 18_4_s & 68.45 & 1025 \\
\hline 18_4_m & 62.13 & 1889 \\
\hline 18_4_1 & 51.87 & 3213 \\
\hline 45_10_s & -18.21 & 4231 \\
\hline 45_10_m & -52.12 & 5421 \\
\hline 45_10_1 & -86.47 & 7521 \\
\hline
\end{tabular}

Table 3. Tabu Search Solutions

\begin{tabular}{|l|l|l|l|l|}
\hline Instance & TS & CPU(ms) & Gap(\%) & $\begin{array}{l}\text { Average } \\
\text { Cost } \\
\text { (FC) }\end{array}$ \\
\hline 18_4_s & 72.15 & 1354 & 5.40 & 68.12 \\
\hline 18_4_m & 65.84 & 1645 & 5.97 & 69.36 \\
\hline 18_4_1 & 58.46 & 2719 & 12.70 & 47.41 \\
\hline 45_10_s & 37.23 & 4215 & 304.44 & 123.95 \\
\hline 45_10_m & 48.56 & 9287 & 193.16 & 31.87 \\
\hline $45 \_10 \_1$ & -56.96 & 19523 & 34.12 & -42.08 \\
\hline $80 \_16 \_s$ & -112.54 & 24569 & & -85.74 \\
\hline $80 \_16 \_m$ & -125.94 & 23694 & & -92.97 \\
\hline $80 \_16 \_1$ & -145.87 & 27964 & & -95.45 \\
\hline
\end{tabular}

Table 4.VNS Solutions

\begin{tabular}{|l|l|l|l|l|}
\hline Instance & VNS & CPU(ms) & Gap(\%) & $\begin{array}{l}\text { Average } \\
\text { Cost } \\
\text { (FC) }\end{array}$ \\
\hline 18_4_s & 71.12 & 1647 & 3.90 & 57.23 \\
\hline 18_4_m & 70.45 & 1941 & 13.39 & 54.87 \\
\hline 18_4_1 & 50.2 & 3012 & 3.21 & 43.98 \\
\hline 45_10_s & 97.69 & 4758 & 636.46 & 118.46 \\
\hline 45_10_m & 31.87 & 9562 & 161.14 & 23.41 \\
\hline 45_10_1 & -51.03 & 22358 & 40.98 & -38.14 \\
\hline 80_16_s & -112.54 & 26417 & & -81.56 \\
\hline $80 \_16 \_m$ & -125.94 & 27874 & & -87.29 \\
\hline $80 \_16 \_1$ & -145.87 & 29451 & & -91.76 \\
\hline
\end{tabular}

The gap percentage value of column 4 of Table 3 and Table 4 is given according to a formula described as follows: $\quad$ Gap $=((($ TS or VNS $)-$ Objective $) \times$ 100) $\div$ Objective.

Where the gap defines the difference in percentage between the quality of the MILP and the two metaheuristics TS and VNS algorithms.

\section{Discussion}

The MILP solutions clearly does not show results for bigger instances, for the purpose of this study, a limit is put to not exceed one hour of execution, afterward the execution stops. As shown from Table 2, the first column infers to instances, the second to the value of the objective function and the third to the CPU running time in milliseconds. The MILP give promising results for smaller instances, but as the instances grow larger, the quality of results becomes degrading and not practical. Therefore, this paper presents the results of the metaheuristics programs by displaying the outcomes of the tabu search (TS) methods and the variable neighborhood search (VNS). As shown from Table 3 and 4 , the first column corresponds to the instances used, the second to the objective function values found either in TS and VNS for each table, the third column shows the running time, where the fourth displays the gap percentage and the last one illustrate the average cost in each of the methods used. From what it shows, the TS and VNS are both good algorithms and displays good results except that the VNS algorithm needs more time to compute because it is considered to be a two stage algorithm and that make him a lot slower than the TS algorithm. Besides that the VNS is capable of producing good quality results as displayed in the Table 4 in comparison with the TS results in Table 3 . The running process is done for a number of times equivalent to 8 so the study displayed the best among all those running process. To summarize, the MILP solutions provided by the linear solver CPLEX produces good quality results but only for small instances, where on larger instances the time of computation increase significantly. For that specific reason, the need to converge to approached methods seems way encouraging. In this paper, the study shows that it can optimize a system based on home health care without considering the total travelled distance because in general and it is very common especially in this field, all papers related to health care use the total travelled distance to make the optimization, but this paper differs from this mentality and opted to proceed differently.

\section{Conclusion and perspectives}

In this paper, we have studied a specific case of the vehicle routing problem in home health care with the fuel consumption reduction aspect to optimize rather 
than the usual and widespread total travel distance. We developed two metaheuristics to show the utility and efficiency of the approached method in comparison with the exact methods especially if the problem in a NP-hard one. This study didn't really dig deep in the fuel consumption aspect because it was not the real purpose rather than to divert future research from getting stuck with the maneuver of exploiting the total travel distance all the time. The limitation of this study is that when applied on larger instances, the algorithms used don't give feasible solution at all. Again, the global warming alarm in the past years until now must in all case encourage us to spend more time in studying aspect that can and will reduce the pollution around us and tempt us to find a way to reduce to the most the pollution around us and around our globe. For future research, we encourage to take the direction of introducing aspect that benefits the environment and not the inverse. As a perspective to this work, analyzing the fuel consumption and involving more criteria minimizing the $\mathrm{CO}_{2}$ emission carbon around us. Another future research line is to involve genetic algorithms and evolutionary algorithms in order to have better results because it involves better search mechanism and are more efficient. A hybridization of two methods is also very interesting to test to see how the results will be.

\section{References}

1. J. Euchi, Do drones have a realistic place in a pandemic fight for delivering medical supplies in healthcare

systemsproblems?Chin.J.Aeronaut.(https://doi.org/1 0.1016/j.cja.2020.06.006) (2020)

2. G.B. Dantzig, J.H. Ramser. The truck dispatching problem. M.Sc, 6(1), 80-91 (1959a)

3. P. Toth, D. Vigo. Vehicle Routing : Problems, Methods, and Applications, SIAM. Vol. 18. (2014)

4. O. Bräysi, W. Dullaert, P. Nakari. The potential of optimization in communal routing problems : case studies from finland. Journal of Transport Geography, 17(6), 484-490 (2009)

5. G.Kindervater, M. Savelsbergh, Vehicle routing: handling edge exchanges. Local search in combinatorial optimization, pp. 337-360 (1997).

6. Y.Nagata, Efficient evolutionary algortihm for the vehicle routing problem with time windows: Edge assembly crossover for the VRPTW. In 2007 IEEE Congress on Evolutionnary Computation (pp. 1175 1182). IEEE Press (2007)

7. S.R. Ait Haddadene, N. Labadie, C. Prodhon. A GRASP $\times$ ILS for the vehicle routing problem with time windows, synchronization and precedence constraints. Expert Syst. Appl, Vol. 66, 274-294 (2016)
8. L.D. Bodin, T. Sexton,. The multi-vehicle subscriber dial-a-ride problem. TIMS studies in M.Sc, 2, 73-86 (1986)

9. J. Desrosiers, Y. Dumas,F. Soumis. A dynamic programming solution of the large-scale singlevehicle dial-a-ride with time windows. American Journal of Mathematical and Management Sciences, 6(3-4), 301-325 (1986)

10. J. Desrosiers, Y. Dumas, M.M. Solomon, F. Soumis. Time constrained routing and scheduling. Handbooks in OR and M.Sc, 8, 35-139 (1995)

11. L.M. Rousseau, M. Gendreau, G. Pesant. The synchronized vehicle dispatching problem. Citeseer (2003)

12. L.M. Rousseau, M. Gendreau, G. Pesant. The Synchronized Dynamic Vehicle Dispatching Problem. INFOR : Information Systems and Operational Research, 51(2), 76-83 (2013)

13. A. Coppi, P. Detti, J. Rafaelli. A planning and routing model for patient transportation in health care. Electronic Notes in Discrete Mathematics, 41, 125-132 (2013)

14. E. Cheng, J.L. Rich. A home health care routing and scheduling problem. Technical report CAAM TR9804, Rice University (1998)

15. S. Bertels, T. Stefan. A hybrid setup for a hybrid scenario : combining heuristics for the home health care problem. Computers \& Operations Research. 33(10), 2866-2890 (2006)

16. P. Eveborn, P. Flisberg,M. Rönnqvist. Laps Care an operational system for staff planning of home care. European Journal of Operational Research, 171(3), 962-976 (2006)

17. A. Trautsamwieser, P.Hirsch. Optimization of daily scheduling for home health care services. Journal of Applied Operational Research, 3(3), 124-136 (2011)

18. C. Akjiratikarl, P. Yenradee, P.R. Drake. PSO-based algorithm for home care worker scheduling in the UK. Computers \& Industrial Engineering, 53(4), 559-583 (2007)

19. K. Doerner, A. Focke, W.J. Gutjahr. Multicriteria tour planning for mobile healthcare facilities in a developing country. European Journal of Operational Research, 179(3), 1078-1096 (2007)

20. D. Bredström, M. Rönnqvist. A branch and price algorithm for the combined vehicle routing and scheduling problem with synchronization constraints. NHH Dept. of Finance Management Science Discussion Paper (2007)

21. D. Bredström, M. Rönnqvist. Combined vehicle routing and scheduling with temporal precedence and synchronization constraints. European Journal of Operational Research, 191(1), 19-31 (2008)

22. R. Redjem, S. Kharraja, X. Xie, E. Marcon. Routing and scheduling of caregivers in home health care with synchronized visits. In $: 9^{\text {th }}$ International 
Conference on Modeling, Optimization \& SIMulation (2012)

23. S. Afifi, D.C. Dang, A. Moukrim. A simulated annealing algorithm for the vehicle routing problem with time windows and synchronization constraints. Pages 259-265: Learning and Intelligent Optimization. Springer (2013)

24. R. Redjem, E. Marcon. Operations management in the home health care services : a heuristic for the caregivers' routing problem. Flexible Services and Manufacturing Journal, 1-24 (2015)

25. N. Labadie, C. Prins, Y. Yang. Iterated local search for a vehicle routing problem with synchronization constraints. Pages 257-263 of : ICORES 2014Proceedings of the $3^{\text {rd }}$ International Conference on Operations Research and Enterprise Systems, Angers, Loire Valley, France (2014)

26. B. Issaoui, I. Zidi, E. Marcon, K. Ghedira. New Multi-Objective Approach for the Home Care Service Problem Based on Scheduling Algorithms and Variable Neighborhood Descent. Electronic Notes in Discrete Mathematics, 47, 181-188 (2015)

27. M. Bazirha, A. Kadrani, \& R. Benmansour. Daily Scheduling and Routing of Home Health Care with Multiple Availability Periods of Patients. Variable Neighborhood Search, 178-193 (2020)

28. R. Liu, X. Xie., V. Augusto, C. Rodriguez. Heuristic algorithms for a vehicle routing problem with simultaneous delivery and pickup and time windows in home health care. European Journal of Operational Research, 230(3), 475-486 (2013)

29. M.Drexl. Synchronization in vehicle routing- A survey of VRPs with multiple synchronization constraints. Transportation Science, 46(3), 297-316 (2012)

30. A. Del Pia, C. Flippi. A variable neighborhood descent algorithm from a real waste collection problem with mobile depots. International Transactions in Operational Research. 13. 125-141 (2006)

31. A. Salazar-aguilar, L.André " The synchronized arc and node routing problem: Application to road marking", Computers \& Operation research, 40(7):1708-1715 (2013)

32. R. Borchani, M. Masmoudi, B. Jarbaoui. Hybrid Genetic Algorithm for Home Health care routing and scheduling problem. CoDIT, 1900-1904 (2019)

33. K. Fagerholt. "Optimal fleet design in ship routing problem", International Transactions in Operational Research, Vol. 6, pp. 453-464 (1999)

34. E. Sambracos, J.A. Paravantis, C.D Tarantilis, C.T. Kiranoudis. "Dispatching of small containers via coastal freight liners: the case of the Aegean Sea", European Journal of Operational Research, Vol. 152, pp. 365-81 (2004)

35. V. Maraš. "Determining optimal transport routes of inland waterway constainer ships", Journal of the
Transportation Research Board, Vol. 2026, pp. 50-8 (2008)

36. O. Apaydin, M.T. Gonollu. "Emission control with route optimization in solid waste collection process: a case study", Sādhāna, Vol. 33. pp.71-82 (2008)

37. G. Taveares, Z. Zaigraiova, V. Semia, M. da Graca Carvalho. "A case study of fuel saving through optimization of MSW transportation routes", Management of Environmental Quality: An International Journal, Vol. 19, pp. 444-54 (2008)

38. T. M.S.Rasmussen, A. Justesen, J. Dohn, J. Larsen. The Home Care Crew Scheduling Problem: Preference-Based Visit Clustering and Temporal Dependencies. European Journal of Operational Research (3), p. 598-610 (2012)

39. D.S Reichmuth, A.E. Lutz, D.K. Manley, J.O. Keller. Comparison of the tehcnical portential for hydrogen battery electric, and conventional lightduty vehicles greenhouses gas emissions and petroleum consumption in the United States. Int. J.Hydrog. Energy 38, 1200-1208 (2013)

40. F.Glover. Tabu Search - Part I. ORSA Journal on Computing 1(3): 190-206 (1989)

41. F. Glover, M. Laguna, R. Marti. Fundamentals of Scatter Search and Path Relinking. Control and Cybernetics 29(3) (1995)

42. F. Glover, M. Laguna. Tabu Search. Springer New York (1993)

43. S. Ichoua, M. Gendreau, J.Y. Potvin. VDTTTT. European Journal of Operational Research 144(2):379-396. (2003) 